\title{
Fabricating multifunctional microbubbles and nanobubbles for concurrent ultrasound and photoacoustic imaging
}

Ruogu Qin, Jeff Xu, Ronald Xu, Chulhong Kim, Lihong V. Wang

Ruogu Qin, Jeff Xu, Ronald Xu, Chulhong Kim, Lihong V. Wang, "Fabricating multifunctional microbubbles and nanobubbles for concurrent ultrasound and photoacoustic imaging," Proc. SPIE 7567, Design and Performance Validation of Phantoms Used in Conjunction with Optical Measurement of Tissue II, 75670L (25 February 2010); doi: $10.1117 / 12.841281$

SPIE. Event: SPIE BiOS, 2010, San Francisco, California, United States 


\title{
Fabricating multifunctional microbubbles and nanobubbles for concurrent ultrasound and photoacoustic imaging
}

\author{
Ruogu Qin ${ }^{1}$, Jeff $\mathrm{Xu}^{1}$, Ronald $\mathrm{Xu}^{{ }^{*}}$, Chulhong Kim${ }^{2}$, Lihong V. Wang ${ }^{2 *}$ \\ ${ }^{1}$ Department of Biomedical Engineering, The Ohio State University, Columbus, OH 43210, ${ }^{2}$ Optical \\ Imaging Laboratory, Department of Biomedical Engineering, Washington University in St. Louis, St. \\ Louis, MO 63130 \\ *Corresponding authors: xu.202@osu.edu (for micro- and nanobubbles) and \\ lhwang@biomed.wustl.edu (for photoacoustic imaging)
}

\begin{abstract}
Background: Clinical ultrasound (US) uses ultrasonic scattering contrast to characterize subcutaneous anatomic structures. Photoacoustic (PA) imaging detects the functional properties of thick biological tissue with high optical contrast. In the case of image-guided cancer ablation therapy, simultaneous US and PA imaging can be useful for intraoperative assessment of tumor boundaries and ablation margins. In this regard, accurate co-registration between imaging modalities and high sensitivity to cancer cells are important.

Methods: We synthesized poly-lactic-co-glycolic acid (PLGA) microbubbles (MBs) and nanobubbles (NBs) encapsulating India ink or indocyanine green (ICG). Multiple tumor simulators were fabricated by entrapping ink MBs or NBs at various concentrations in gelatin phantoms for simultaneous US and PA imaging. MBs and NBs were also conjugated with CC49 antibody to target TAG-72, a human glycoprotein complex expressed in many epithelial-derived cancers.

Results: Accurate co-registration and intensity correlation were observed in US and PA images of MB and NB tumor simulators. MBs and NBs conjugating with CC49 effectively bound with over-expressed TAG-72 in LS174T colon cancer cell cultures. ICG was also encapsulated in MBs and NBs for the potential to integrate US, PA, and fluorescence imaging.
\end{abstract}

Conclusions: Multifunctional MBs and NBs can be potentially used as a general contrast agent for multimodal intraoperative imaging of tumor boundaries and therapeutic margins.

Keywords: Ultrasound, photoacoustic, intraoperative, microbubbles, nanobubbles, contrast agent, cancer ablation

\section{INTRODUCTION}

Thermal ablation processes destroy tumor tissue by localized deposition of thermal energy that causes protein denaturation and coagulation necrosis ${ }^{[1]}$. They hold the promise of less invasive cancer management with minimal trauma, outpatient treatment, and fast recovery. However, broad clinical acceptance of many cancer ablation processes is hindered by controversial issues and concerns related with clinical safety, efficacy, and long-term local recurrence. One major limitation is the lack of intraoperative assessment of tumor boundaries and ablation margins ${ }^{[2]}$. Clinical ultrasound (US) has been used for tumor localization and ablation probe placement in a cancer ablation procedure ${ }^{[3]}$. However, US alone is not appropriate for intraoperative imaging because of its low specificity for soft tissue mass and its susceptibility to ablation-induced gas bubbles. In this regard, photoacoustic (PA) imaging may provide complementary information regarding tumor functional characteristics and ablation margins. Integrating US and PA into a single handheld imager will take advantages of both modalities without introducing too much hardware complexity. However, accurate calibration and co-registration between these two modalities is difficult because of the mismatch between tissue 
structural and functional heterogeneities. A dual-mode imaging agent with simultaneously strong US and PA contrasts will not only help to calibrate the US-PA hybrid imaging system but also provide imaging markers for tumor boundary and therapeutic margin assessment with multiple imaging modalities. This paper describes our recent effort to encapsulate India ink in biodegradable microbubbles (MBs) and nanobubbles (NBs) for concurrent US and PA imaging.

\section{FABRICATING DUAL-MODE MBS AND NBS}

PLGA (50:50, RG 502H 12000 Da MW, Boehringer Ingelheim) MBs and NBs encapsulating India ink were fabricated with a modified double emulsion process ${ }^{[4]}$. Firstly, four solutions were prepared: (1) $5 \mathrm{~mL}$ of $2.5 \% \mathrm{w} / \mathrm{v}$ PLGA solution of methylene chloride (CH2Cl2, Fisher Scientific), (2) $50 \mathrm{~mL}$ of $4 \% \mathrm{w} / \mathrm{v}$ polyvinyl alcohol (PVA, Fisher Scientific) aqueous solution, (3) $0.5 \mathrm{~mL}$ of $50 \% \mathrm{v} / \mathrm{v}$ ink (Higgins Fountain Pen India non-waterproof black ink, Sanford) and $4 \%$ w/v PVA aqueous solution, and (4) $100 \mathrm{~mL}$ of $5 \% \mathrm{v} / \mathrm{v}$ isopropanol aqueous solution. Secondly, the $0.5 \mathrm{~mL}$ aqueous solution of ink and PVA was added to the $5 \mathrm{~mL}$ PLGA solution of $\mathrm{CH} 2 \mathrm{Cl} 2$, and emulsified in an ice bath. Then, the emulsion was added drop-wise with a $1000 \mu \mathrm{L}$ pipette to the $50 \mathrm{~mL}$ PVA solution in an ice bath and emulsified again. The double emulsion was then added to the isopropanol solution, stirred for 1.5 hours by a magnetic stirrer, and then centrifuged. After centrifugation, the supernatant was discarded, and the bubble precipitate was washed by deionized water. The process of centrifugation and washing was repeated three times. The washed spheres were then freeze-dried by a Lyph-lock 4.5 freeze dry system (Labcono Corp.) for 36 hours. Dried bubbles were harvested and stored in a glass bottle at $0{ }^{\circ} \mathrm{C}$ for further use. For the MBs, the first and second emulsion steps were conducted with a model 17105 Omni Mixer homogenizer (Omini International) at 20,000 rpm for 5 minutes and 10,000 rpm for 3 minutes, respectively. The centrifugation was carried out with a Centrifuge 5810R (Eppendorf) at $1500 \mathrm{rpm}$ for 10 minutes. For the NBs, the first and the second emulsion steps were conducted with an Omni-Ruptor 250 ultrasound probe (Omini International) at $90 \mathrm{~W}$ for 2 minutes and at $30 \mathrm{~W}$ for one minute, respectively. The centrifugation was carried out with a SORVALL RC5B high speed centrifuge at $9000 \mathrm{rpm}$ for 10 minutes. Figure 1(a) shows the cross-section of a MB or NB. The solid PLGA shell encapsulates india ink and air inside, forming a stable spherical bubble. The average size of MBs is $1.01 \pm$ $0.73 \mu \mathrm{m}$, and that of NBs is $0.29 \pm 0.09 \mu \mathrm{m}$. To fabricate dual-mode tissue simulating phantoms, we first mixed gelatin powder (Great Lakes Gelatin, grayslake, IL) with warm water $\left(\sim 45^{\circ} \mathrm{C}\right)$. The mixture was then degassed, forming $0.15 \mathrm{mg} / \mathrm{mL}$ clear gelatin solution. Eight cylindrical tumor simulators were fabricated by dispersing four concentrations $(2.5,5,10,15 \mathrm{mg} / \mathrm{mL})$ of MBs or NBs respectively into above clear gelatin solution. The tumor simulators were cast into a gelatin pad $(3 \mathrm{~cm} \times 4 \mathrm{~cm} \times 0.5 \mathrm{~cm})$.

\section{DUAL MODE US AND PA IMAGING}

The tissue simulating phantom was imaged by a PA macroscope and a $5 \mathrm{MHz}$ US probe. For PA imaging, the mechanism of the ring-shaped light illumination is described fully in reference ${ }^{[5]}$. The schematic of the system is showed in Figure 1. To irradiate the phantoms, we used the wavelength-tunable Ti-Sapphire laser (LT-2211A, LOTIS TII), pumped by a Q-switched Nd:YAG laser (LS-2137, LOTIS) with a pulse duration of $6.5 \mathrm{~ns}$ and a pulse repetition rate of $10 \mathrm{~Hz}$. The light fluence on the phantom surface was $3.4 \mathrm{~mJ} / \mathrm{cm}^{2}$, within the current ANSI limit ${ }^{[6]}$. A donut-shaped light $(\lambda=767$ $\mathrm{nm}$ ), formed by concave and conical lenses, traveled through an optical condenser, and was coaxially focused with the ultrasound focal zone in water. The phantoms were positioned under a water tank that had a window with a transparent thin membrane. The PA waves, detected by a single-element 5-MHz ultrasound transducer (V308, Panametrics-NDT), were first amplified and then transferred to an oscilloscope. The axial and lateral resolutions were 138 and $490 \mu \mathrm{m}$, respectively. One-dimensional depth-sensitive images along the z-axis (referred to as A-lines) were obtained by measuring the times of arrival of the PA signals. By scanning the samples along the $\mathrm{x}$ and $\mathrm{y}$ directions, three-dimensional PA images were formed. Three-dimensional images were projected into two-dimensional images through maximum amplitude projection (MAP), which projected the strongest signal along each A-line onto the corresponding $\mathrm{x}-\mathrm{y}$ plane. For US imaging, the phantom is bathed in cool water and US signal is acquired from the side. Beam focus is set to $\sim 1.6 \mathrm{~cm}$ which is about half the traveling distance of US wave through the phantom. Time gain control (TGC) is default to provide comparable contrast for each imaging slide. 


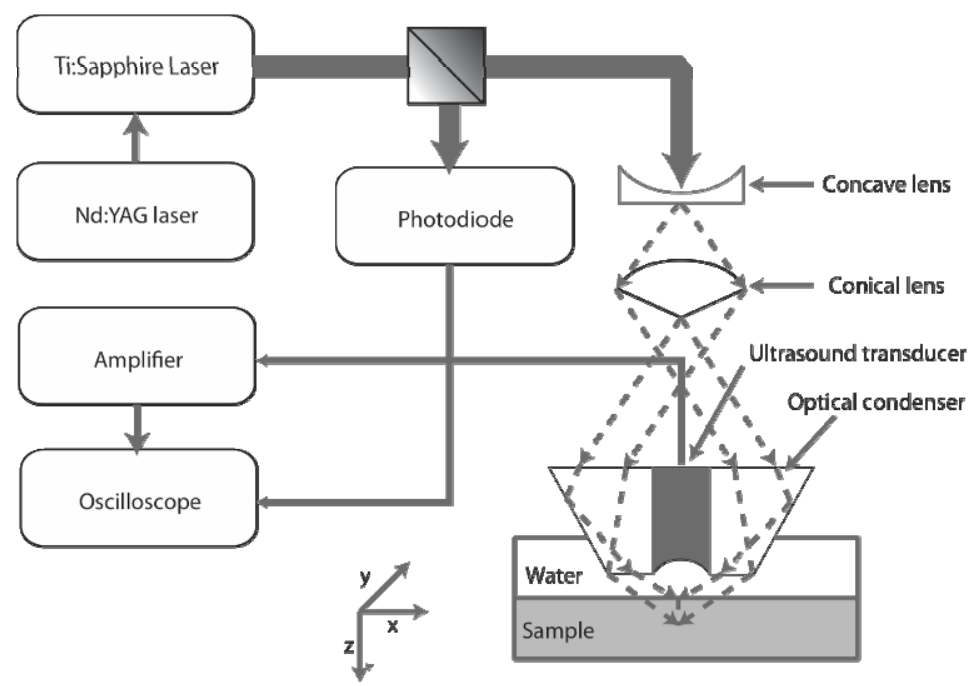

Figure 1. Schematic of the photoacoustic macroscope

Figures 2(a) to 2(c) show the photographic, PA, and US images acquired from a tissue simulating phantom as described above. The tissue simulating phantom embedded 8 tumor simulators with different MB and NB concentrations. Tumor simulators \#1 to \#4 dispersed MBs at concentration levels of $2.5,5,10,15 \mathrm{mg} / \mathrm{mL}$, respectively. Tumor simulators \#5 to \#8 dispersed NBs at concentration levels of $2.5,5,10,15 \mathrm{mg} / \mathrm{mL}$, respectively. Figure 2(d) shows the correlation between the PA signal strength and the MB/NB concentration. Figure 2(c) shows the correlation between the US contrast and the MB/NB concentration. Both MBs and NBs show PA and US contrasts. 2.5 and $5.0 \mathrm{mg} / \mathrm{mL}$ MBs are not quite clearly visualized in US image, the rest of the tumor simulators can be clearly discerned. The NBs seem to be better visible than MBs in US. While the mechanism for acoustic contrast enhancement by MBs has been reviewed in ${ }^{[7]}$, the mechanism for NBs is still not quite clear. A possible explanation is the aggregation and layering effect of NBs ${ }^{[7-9]}$. The above results show that by encapsulating India ink into MBs or NBs, it is possible to provide dual-mode contrast for PA and US imaging.

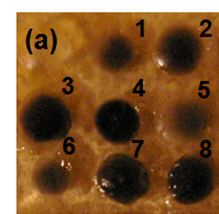

$5 \overline{\mathrm{mm}}$

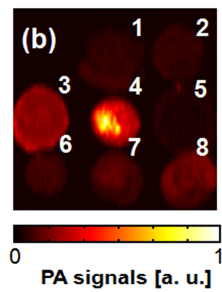

PA signals [a. u.]
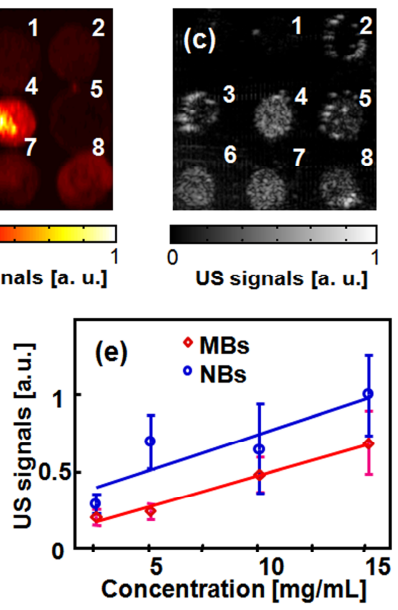

Figure 2. a) A photo of the tissue simulating phantom; (b) PAT image of the phantom; (c) US image of the phantom; The number 1 through 4 correspond to $2.5,5,10,15 \mathrm{mg} / \mathrm{mL}$ MBs and 5 through 8 correspond to $2.5,5,10,15 \mathrm{mg} / \mathrm{mL} \mathrm{NBs}$; (d) quantitative comparison of PAT signal; (e) quantitative comparison of US signal.

We added chicken breast tissues atop the phantom to increase imaging depth. With a 1-cm thick layer of chicken breast tissue, all eight targets are clearly shown in Figure 3. 


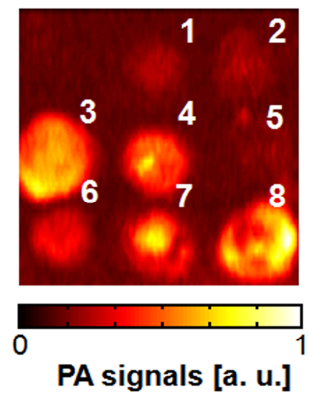

Figure 3. PAT image of the phantom positioned below $1 \mathrm{~cm}$ of chicken breast tissues.

\section{CONJUGATING MBS AND NBS FOR CANCER TARGETING}

TAG-72 is a human glycoprotein complex expressed in many epithelial-derived cancers, including colorectal, pancreatic, breast, ovarian, and gastric cancers ${ }^{[10]}$. Anti-TAG-72 antibodies, such as murine B72.3, murine CC49, and humanized HuCC $49 \Delta \mathrm{C}_{\mathrm{H}} 2$, have been used to target over-expressed TAG-72 in both cancer xenograft models and tissue samples from cancer patients ${ }^{[11,12]}$. In the past, we have conjugated murine $\mathrm{CC} 49$ and $\mathrm{HuCC} 49 \Delta \mathrm{C}_{\mathrm{H}} 2$ with Cy7, an ICG derivative, for near infrared fluorescence imaging on LS174T colorectal cancer xenograft nude mice ${ }^{[13]}$. Our animal experiments demonstrated that $\mathrm{Cy} 7$ labelled CC49 and $\mathrm{HuCC} 49 \Delta \mathrm{C}_{\mathrm{H}} 2$ are able to target tumors in living colorectal cancer xenograft mice for near infrared fluorescence imaging of tumor boundaries. We also conjugated $\mathrm{HuCC}_{4} \mathrm{~A} \Delta \mathrm{C}_{\mathrm{H}} 2$ antibody with MBs and NBs for cancer targeting and imaging ${ }^{[14]}$. Our binding tests on LS174T cancer cell culture showed that antibody conjugated MBs and NBs can effectively bind with cancer cells, while bare MBs and NBs do not bind with cancer cell well.

\section{CONCLUSION AND FUTURE WORK}

We have successfully demonstrated the dual-mode contrasts of MBs and NBs encapsulating India ink. By conjugating MBs and NBs with cancer-targeting antibodies and by encapsulating India ink, we may produce novel contrast agents for simultaneous US and PA imaging of tumor boundaries as well as ablation margins. It is also possible to encapsulate many other imaging agents into MBs and NBs for multimodal imaging. For example, Gadolinium can be encapsulated for Magnetic Resonance Imaging. MBs and NBs can be radiolabelled for PET-CT imaging. We expect that such multimodal MB and NB agents will provide disease-specific contrasts for intraoperative tumor assessment.

\section{ACKNOWLEDGEMENT}

This work was supported in part by grants from the National Institutes of Health (R01 EB000712, R01 EB008085, and U54 CA136398 - the Network for Translational Research - to L.V.W.) and the Department of Defense Breast Cancer Research Program (W81XWH-07 to R. X.). L.V.W. has a financial interest in Microphotoacoustics, Inc. and in Endra, Inc., which, however, did not support this work.

\section{REFERENCE}

[1] C. J. Diederich, "Thermal ablation and high-temperature thermal therapy: overview of technology and clinical implementation," Int J Hyperthermia, 21(8), 745-53 (2005).

[2] CIGNA, [Radiofrequency ablation for breast cancer], (2008).

[3] M. Tonolini, and L. Solbiati, [Ultrasound imaging in tumor ablation] Springer, New York(2005).

[4] R. X. Xu, J. Huang, J. S. Xu et al., "Fabrication of indocyanine green encapsulated biodegradable microbubbles for structural and functional imaging of cancer," J Biomed Opt, 14(3), 034020 (2009).

[5] K. H. Song, and L. V. Wang, "Deep reflection-mode photoacoustic imaging of biological tissue," J Biomed Opt, 12(6), 060503 (2007). 
[6] , [American national standard for safe use of lasers (ANSI Z136.1-2000)] Laser Institute of America, (2000).

[7] E. Stride, and N. Saffari, "Microbubble ultrasound contrast agents: a review," Proceedings of the Institution of Mechanical Engineers, Part H: Journal of Engineering in Medicine, 217(6), 429-447 (2003).

[8] J. Liu, J. Li, T. J. Rosol et al., "Biodegradable nanoparticles for targeted ultrasound imaging of breast cancer cells in vitro," Physics in medicine and biology, 52(16), 4739-4748 (2007).

[9] J. N. Marsh, K. C. Partlow, D. R. Abendschein et al., "Molecular imaging with targeted perfluorocarbon nanoparticles: Quantification of the concentration dependence of contrast enhancement for binding to sparse cellular epitopes," Ultrasound in medicine \& biology, 33(6), 950-958 (2007).

[10] V. G. Johnson, J. Schlom, A. J. Paterson et al., "Analysis of a human tumor-associated glycoprotein (TAG-72) identified by monoclonal antibody B72.3," Cancer Res, 46(2), 850-7 (1986).

[11] S. C. Lottich, W. W. Johnston, C. A. Szpak et al., "Tumor-associated antigen TAG-72: correlation of expression in primary and metastatic breast carcinoma lesions," Breast Cancer Res Treat, 6(1), 49-56 (1985).

[12] D. C. Slavin-Chiorini, S. V. Kashmiri, H. S. Lee et al., "A CDR-grafted (humanized) domain-deleted antitumor antibody," Cancer Biother Radiopharm, 12(5), 305-16 (1997).

[13] P. Zou, S. Xu, A. Wang et al., "Near-Infrared Fluorescence Labeled Anti-TAG-72 Monoclonal Antibodies for Tumor Imaging in Colorectal Cancer Xenograft Mice," Molecular Pharmacuetics, 6(2), 428-440 (2009).

[14] J. S. Xu, J. Huang, R. Qin et al., "Synthesizing and binding dual-mode poly (lactic-co-glycolic acid) (PLGA) nanobubbles for cancer targeting and imaging," Biomaterials(doi:10.1016/j.biomaterials.2009.11.052), (2009). 